\title{
TEORIA DOS JOGOS: CONCEITOS, FORMALIZAÇÃO MATEMÁTICA E APLICAÇÃO À DISTRIBUIÇÃO DE CUSTO CONJUNTO
}

\author{
Reginaldo Santana Figueiredo \\ Prof. Dr. do Departamento de Engenharia de Produção \\ Universidade Federal de São Carlos
}

O presente trabalho tem por objetivo a sistematização e a apresentação dos conceitos fundamentais da Teoria dos Jogos, a formalização matemática dos principais conceitos clássicos de solução de um jogo e ilustrar sua aplicação ao problema da distribuição de custo conjunto. Como produto de uma pesquisa de alguns anos, conseguimos reunir sistematicamente o conjunto de conceitos de solução de jogos donde se derivam todos conceitos encontrados nos textos atuais. Apresentamos os conceitos de solução para os chamados jogos cooperativos através do conceito de objeção e contra-objeção de forma a mostrar que os mesmos podem ser vistos como um processo de negociação.

Palavras-chaves: decisões interdependentes, modelagem de conflito, jogo, modelagem matemática..

\section{Introdução}

A Teoria dos Jogos, a qual poderia se chamar muito apropriadamente de Teoria das Decisões Interdependentes, tem como objeto de análise situações onde o resultado da ação de indivíduos, grupo de indivíduos, ou instituições, depende substancialmente das ações dos outros envolvidos. Em outras palavras, trata de situações onde nenhum indi-víduo pode convenientemente tomar decisão sem levar em conta as possíveis decisões dos outros.

O primeiro passo para o seu desenvolvimento foi dado por von Neumann, em 1928, com a demonstração do teorema minimax. Contudo, só chamou a atenção do público em 1944, quando em parceria com Morgenstern, propôs a análise do comportamento econômico via perspectiva do "jogo de estratégia" criando assim a expectativa de reformulação da teoria econômica numa base totalmente nova, na qual o conceito de "processo competitivo" seria reestruturado em termos de mecanismos onde os agentes econômicos atuam estrategicamente (von NEUMANN \& MORGENSTERN, 1972).

Neste primeiro momento, as aplicações mais bem sucedidas se realizaram no campo da análise de mercados oligopolistas, onde obtiveram resultados interessantes, contudo ainda limitados pelos recursos matemáticos específicos disponíveis. 
Na década de 50 surgem novos horizontes. Em 1950, John F. Nash demonstra o teorema minimax para grandes números de agentes. Em 1952, Lloyd Shapley apresenta o conceito de "núcleo".E em 1959, Martin Shubik demonstra que a clássica "curva de contrato" de Edgeworth era idêntica ao conceito de solução desenvolvido por Shapley permitindo a teoria neoclássica se livrar de um dos seus principais problemas metodológicos. Naquele momento, enquanto a análise do problema do equilíbrio geral, conduzida principalmente por Arrow, Debreu e McKenzie, era estritamente paramétrica, ao considerar os agentes econômicos como "tomadores de preços", a Teoria dos Jogos, de posse de novos recursos técnicos, permitia analisar a questão da formação de preços como produto de um amplo processo de barganha multilateral.

Nos anos 60 a popularidade da teoria entrou novamente em estado latente. Seu debate ficou restrito apenas a alguns pequenos grupos de pesquisadores desmotivados a publicar os resultados de suas pesquisas. Esse fenômeno já havia, de certa forma acontecido nos anos 50, quando por alguns anos tornou-se praxe publicar apenas partes dos resultados das pesquisas realizadas. Os resultados foram muito pouco divulgados. Um exemplo importante foi o Folk Theorem que, devido a esse tipo de comportamento, ficou praticamente desconhecido por um período de tempo considerável.

A partir da segunda metade do anos 60, engenheiros e economistas começaram a perceber a Teoria dos Jogos como um instrumento de considerável alcance para uma ve-lha questão que voltara a tomar fôlego: a análise, projeto e implementação de mecanis-mos de alocação de recursos. O principal protagonista desta questão foi HURVICZ (1973). Sua preocupação central estava voltada para a análise institucional, especial-mente em economias informacionalmente descentralizadas. Para tal propósito, se envol-veu na construção de mecanismos de alocação ou de planejamento que produzissem re-sultados "satisfatórios". Como cada mecanismo de alocação de recursos contém implici-tamente definido um jogo, abre-se assim um novo campo de pesquisa: a análise e proje-tos de mecanismos de alocação de recursos através das técnicas da Teoria dos Jogos.

A literatura concernente à teoria da escolha social também acabou se constituindo em uma fonte de pesquisa para a Teoria dos Jogos. Este campo de pesquisa teve origem quando GIBBARD (1973) e SATHERHWAITE (1975), independentemente, resolve-ram indagar o que aconteceria se os agentes estudados por ARROW (1965) votassem estrategicamente ou de uma forma que não fosse isomórfica em relação às suas verdadeiras preferências. A resposta encontrada foi, como era de se esperar, que as regras de votação usualmente utilizadas podem permitir escolhas sociais que não sejam "ótimos de Pareto". A análise desta questão acabou gerando um campo de pesquisa onde se procura desenvolver jogos que possam representar mecanismos de escolha, onde os agentes envolvidos são incentivados a votarem estrategicamente.

Outro campo de pesquisa também desenvolvido na década de $70 \mathrm{diz}$ respeito à distribuição de custo conjunto. A Teoria dos Jogos tem se dedicado de forma brilhante também a essa questão e tem se destacado ao analisar e propor soluções para situações concretas e como as que serão apresentadas adiante. 
Cabe ressaltar que a análise do equilíbrio geral desenvolvida pela Teoria Neoclássica tem sido suplantada quase completamente pelo Teoria de Jogos nãocooperativos, principalmente a partir da segunda metade década de 80 , quando a atenção de muitos pesquisadores tais como, por exemplo, JACQUEMIN (1987), TIROLE (1988), FARREL \& MASKIN, (1989), KREPS (1990) e FUDENBERG (1991), vêm sendo dirigida para modelagem de jogos dinâmicos, levando em conta a hipótese de informação imperfeita e de informação incompleta. A possibilidade das firmas fazerem coalizões, em modelos dinâmicos, tem se mostrado maior que em modelos estáticos e oferecido razoáveis subsídios para estabelecimento de política antitruste.

Dada a necessidade de entendermos melhor a amplitude das aplicações que citamos acima, as quais em sua maioria serão detalhadas em FIGUEIREDO (1993), vejamos como ela pode ser formalizada.

Um jogo pode ser exposto matematicamente de diversas maneiras, isto é, de acordo com as propriedades dele que desejamos explorar. Nosso objetivo aqui é darlhe a expressão mais geral possível sem deixar escapar as suas propriedades mais essenciais.

A forma mais detalhada de se apresentar um jogo é na forma extensiva a qual se refere à descrição concentrada no movimento seqüencial do mesmo. Nessa forma as decisões são tomadas uma após a outra. O conceito de estratégia tomado como a descrição completa de como uma pessoa que participa de um jogo pode agir sob quaisquer circunstâncias, ou um curso de ação qualquer de um agente em um jogo na forma extensiva, nos permite definir e expressar o jogo em uma forma mais simples e objetiva, e por isso de maior importância teórica, chamada forma normal ou forma estratégica. Podemos expressar um jogo na forma normal utilizando apenas as estratégias disponíveis para cada jogador e os respectivos resultados associados a cada elemento do conjunto constituído do produto cartesiano dos conjuntos de estratégias individuais. Podemos dizer ainda que um jogo está na forma normal quando toda a seqüência de decisões que devem ser tomadas enquanto ele se processa na forma extensiva pode ser reunida em uma única e particular decisão para o jogador: a escolha de uma estratégia. Todo jogo na forma extensiva pode ser expresso na forma normal (LUCE \& RAIFFA, 1957, pag.59). Representa-se o jogo na forma extensiva só quando se faz necessário, para seu tratamento teórico, o conhecimento de certas propriedades que estão associadas ao movimento seqüencial do processo de tomada de decisão.

Muitas vezes o tratamento da questão em análise exige muito menos informação do que as apresentadas pela forma normal, necessitando apenas o conhecimento do conjunto de resultados que cada jogador ou coligação de jogadores pode garantir para eles próprios, se eles agem como equipe, independentemente do que o restante dos jogadores possa fazer contra eles. Quando representamos o jogo fazendo uso apenas dessas informações dizemos que o mesmo está na forma de função característica.

Um jogo também pode se diferenciar de acordo com o conjunto de informações que os jogadores possuem. Um jogo é de informação completa ou de informação incom-pleta se cada jogador conhece ou não as seguintes informações: (a) o conjunto de joga-dores; (b) as estratégias disponíveis para cada jogador; e (c) todos possíveis 
resul-tados para todos jogadores. Um jogo é de informação completa quando cada jogador conhece (a), (b) e (c) e é informação de incompleta quando um ou mais jogadores desconhecem alguma das informações citadas. Em relação aos lances ou movimentos, um jogo pode ser de informação perfeita e de informação imperfeita. Um jogo é de informação per-feita se a cada movimento todos os jogadores conhecem as escolhas feitas nos movimen-tos anteriores. Caso esta condição não ocorra, o jogo é de informação imperfeita.

Uma situação para ser considerada como jogo, teria que apresentar a existência de conflito e interdependência entre as decisões dos participante. Esta é a caracterização mais abstrata que podemos fazer de um jogo. No entanto, num plano mais concreto, podemos identificar dois tipos de jogo: (1) o jogo não-cooperativo, quando as condições orgânicas do mesmo não permitem a formação de coalizões que possam determinar o resultado do jogo, e (2) o jogo cooperativo, quando as próprias condições orgânicas do jogo permitem a possibilidade dos participantes atuarem por meio de coalizões.

\section{Jogos não-cooperativos}

Para caracterizarmos o que entendemos por conceito de solução para um jogo não-cooperativo suponhamos uma situação dada por: (1) a existência de um conjunto de agentes, com $\mathbf{n}$ jogadores, $\mathbf{I}=\{1, \ldots, \mathbf{n}\}$; (2) uma família de conjuntos de estratégias $\left(S_{i}\right)_{i \in I}$, onde cada conjunto $S_{i}$ representa todas estratégias $S_{f}$ disponíveis para cada jogador $\mathbf{i}$, (i I I); (3) uma família de funções $\left(P_{\mathbf{i}}\right)_{\mathbf{i} \in \mathrm{I}}$, de valor real, onde cada uma define um resultado ou "payoff" $P_{i}(\mathbf{s})$ para cada jogador em função das decisões tomadas por todos jogadores, ou em função do vetor que contém as estratégias tomadas por cada jogador, $s=\left(\boldsymbol{S}_{1}, \ldots, \boldsymbol{S}_{1}, \ldots, \boldsymbol{S}_{\mathbf{S}}\right)$, onde, por sua vez, o número real $\mathrm{P}_{\mathrm{i}}(\mathbf{s})$ pode se visto como utilidade do resultado do jogo para o indivíduo $\mathbf{i}$, quando todos escolheram suas estratégias definindo assim o vetor $\mathbf{s}$.

Na situação descrita acima a questão do indivíduo é escolher a sua estratégia $S_{f}$, supondo um certo comportamento dos outros participantes do jogo na escolha de suas respectivas estratégias, de forma a maximizar o seu ganho, ou a sua função utilidade; em outras palavras tirar o máximo proveito do jogo. Matematicamente, se representarmos por $\mathbf{s} \backslash S_{\sharp}$ a ênupla de estratégias onde a i-ésima componente do vetor $\mathrm{S}$ foi substituída por $S_{\sharp}$, podemos definir a solução do problema do jogador individual como $S_{H}$, tal que:

$$
P_{i}\left(\mathbf{s} \backslash S_{f}\right) \quad P_{i}(s) \text { para todo } S_{t} \quad S_{i} \text { e } \mathbf{i}
$$

A questão para o analista, aqui, é responder, sob que condições, o jogo terá solução. Concebendo solução como uma situação onde, dadas as decisões, nenhum agente tem qualquer estímulo para altera-la. Podemos dizer que, matematicamente, existirá solução se existir uma combinação de estratégias $\mathbf{s}^{*}$ para a qual

$$
\mathrm{P}_{\mathrm{i}}\left(\mathbf{s}^{*}\right) \quad \mathrm{P}_{\mathrm{i}}\left(\mathbf{s}^{*} \backslash \boldsymbol{S}_{\sharp}\right) \text { para todo } \boldsymbol{S}_{+} \quad \mathrm{S}_{\mathrm{i}} \text { e } \mathbf{i}
$$


A ênupla de estratégias $\mathbf{s}^{*}$ representa o equilíbrio de um jogo não-cooperativo ou ponto de equilíbrio de Nash. Esse conceito de solução implica que nenhum participante se beneficia mudando sua estratégia em $\mathbf{s}^{*}$, quando todos os demais mantêm as suas (NASH, 1951). Em outras palavras, no equilíbrio, nenhum agente tem estímulo para alterar unilateralmente a sua estratégia.

Para falarmos precisamente de um conceito de solução para um jogo nãocooperativo de n-pessoas convém ainda separarmos os jogos finitos dos jogos infinitos. Jogos finitos são aqueles onde cada participante se depara com um conjunto finito de escolhas, ou seja, eles escolhem suas estratégias dentro de um conjunto finito de alternativas. No jogo infinito o conjunto de alternativas, onde cada participante faz sua escolha, é infinito.

Solução para um jogo infinito: o teorema de Nikaido e Isoda demonstrou (SCHOTTER \& SCHWÖDIAUER, 1980) que, dado um jogo na forma normal, se o conjunto de estratégias dos jogadores consiste de subconjuntos convexos e compactos de espaços Euclidianos, $S_{i} \subset \Re^{m i}$, e suas funções de resultados, $P_{i}$, forem contínuas e côncavas em $\mathrm{S}_{\mathrm{i}}$, existirá sempre um ponto de equilíbrio na forma em que foi definido por Nash. Mas, no entanto, não será sempre necessariamente único.

Solução para um jogo finito: nesse caso, sob domínio de estratégias puras, ou seja sob o domínio de estratégias na forma definida até aqui, não é possível estabelecer condições gerais sob as quais um jogo não-cooperativo de n-pessoas teria sempre solução. Todavia o teorema principal de NASH (1951) mostra que sob o domínio de estratégias mistas, estratégias puras associadas a uma distribuição de probabilidade, todo jogo finito tem no mínimo um ponto de equilíbrio. Convém acrescentar que, como no caso do teorema aplicado aos jogos infinitos, o teorema que sustenta a solução para os jogos finitos, além de não garantir a unicidade, não garante também a propriedade de equivalência e intercambialidade quando o problema apresenta mais de uma solução

Portanto, sob a condição de impossibilidade dos agentes estabelecerem coalizões nem mesmo para o caso mais simples, que é o jogo de duas pessoas do tipo somazero, ou soma-constante, dado que todo jogo de soma-constante pode ser transformado em um jogo de soma-zero, pode se garantir sempre a existência de pontos de equilíbrio com estratégias puras, e quando esses pontos existem não são necessariamente únicos. No entanto, adotando-se o conceito de estratégias mistas é possível construir uma teoria geral para os jogos de duas pessoas do tipo soma-zero. $\mathrm{O}$ fundamento dessa generalização foi fornecido por von Neumann através do teorema minimax, segundo o qual sob o domínio de estratégias mistas todo jogo de duas pessoas do tipo soma-zero tem no mínimo um ponto de equilíbrio e quando possui vários eles são equivalentes e as respectivas estratégias de equilíbrios são intercambiáveis. Nesse caso as estratégias mistas que reproduzem a solução de Nash podem ser calculadas através do método simplex aplicado ao problema de programação linear formalizado a partir dos parâmetros fornecido pelo respectivo jogo. 


\section{Jogos cooperativos}

Os jogos chamados cooperativos constituem uma classe determinada de jogos que se diferenciam dos jogos não-cooperativos principalmente pelo fato de que, ao contrário dos jogos não-cooperativos, eles possuem, como já afirmamos anteriormente, em sua estrutura interna, condições que favorecem a possibilidade dos agentes fazerem coalizões entre si com vistas a garantir um determinado resultado. Por uma coalizão entendemos qualquer subconjunto do conjunto de jogadores, I, constituído de jogadores que resolvem agir como uma equipe no processo de escolha de estratégias.

A análise do jogo cooperativo dispensa a utilização do jogo na forma normal, porque ela necessita apenas dos resultados (payoffs) ou ganhos que os jogadores ou as coalizões podem garantir para si. Essas informações estão contidas na função característica.

A função característica, $\mathbf{V}$, é definida como a relação que associa a cada coalizão $\mathbf{K} \subset \mathrm{I},(\mathbf{K} \neq \phi)$, o conjunto de todos vetores-resultados ou vetores de ganhos, $\mathrm{V}(\mathrm{K}) \subset \mathfrak{R}^{\mathrm{k}}$ (espaço Euclidiano de dimensão k, onde $\mathbf{k}$ é o número de elementos da coalizão K) que a coalizão K pode garantir. Na verdade, dado o nosso propósito, não precisamos, como veremos adiante, utilizar ou conhecer extensivamente a função característica. Para efeito de clareza e simplicidade suporemos que a função de resultados que represente os ganhos individuais de cada participante do jogo será "transferível". Assumir que a função de resultados é transferível significa que o ganho total alcançado por uma coalizão pode livremente ser dividido entre seus membros da forma que melhor lhes convier. Podemos assim adimitir a existência de pagamentos à parte, ou ainda, em outras palavras, admitir a possibilidade de redistribuição dos ganhos fora do jogo propriamente dito. E para tanto basta que a função de resultados sejam transferível, isto é, basta que o valor do resultado seja mantido quando redistribuido.

Na análise da Teoria dos Jogos, sob a hipótese de que as funções de resultados sejam transferíveis, não existe perda conceitual. Todos os conceitos de solução para jogos cooperativos não dependem da hipótese de que as funções de resultados sejam transferíveis ou não (SHUBIK,1987, pag.369)

O uso da hipótese de transferêcia de utilidade é uma forma de simplificar o estudo dos jogos cooperativos sem perda dos aspectos relevantes do jogo, como mostram LUCE \& RAIFFA (1957), FRIEDMAN (1986). Esta propriedade nos permite trabalhar com o o valor máximo alcançável pela soma de utilidades dos participantes de uma coalizão K. Dessa forma a função característica será representada por um único valor $\mathrm{v}(\mathrm{K})$, sem nos preocuparmos com outros possíveis valores que ela poderia alcançar. Nos interessa apenas o valor máximo que a coalizão pode conseguir, o qual será redistribuído através de pagamentos feitos à parte.

Redefiniremos a função característica de um jogo de n-pessoas, como: um valor real definido nos subconjuntos de $\mathbf{I}$, que atribui a cada $\mathrm{K} \subset \mathrm{I}$ o máximo valor (para $\mathrm{K}$ ) do "jogo de duas pessoas" realizado entre $\mathrm{K}$ e $\mathbf{I}-\mathrm{K}$, supondo que essas duas coalizões se formam. Portanto, daqui por diante, $v(K)$ representa o máximo valor que os 
membros de K podem obter do jogo, independente do que o restante dos participantes possa fazer. Donde, por razões óbvias, segue que:

$$
v(\phi)=0
$$

e ainda se K e L são duas coalizões disjuntas, só unirão suas forças, logicamente, se conseguirem no mínimo o que poderiam ganhar se agissem separadamente. Logo a função característica $v(K)$, deve obedecer a uma propriedade chamada superaditividade, isto é,

$$
v(K \cup L) \geq v(K)+v(L) \text { se } K \cap L=\phi
$$

O conceito de função característica é de crucial importância para a análise de solução para os jogos cooperativos. Essa categoria de jogo, ao contrário da dos jogos não-cooperativos, fornece um espaço extenso e propício para uma gama de diferentes conceitos de solução. Por essa razão apresentaremos apenas aqueles jogos considerados mais importantes pela literatura especializada no assunto.

Dada a propriedade de superaditividade da função característica $v$, é razoável se supor que a grande coalizão, ou seja, a coalizão de todos elementos do conjunto I, se formará. Dessa forma, portanto, a questão que resta será como o valor de v(I) se distribuirá entre os participantes de maneira que essa distribuição seja estável. Para analisar essa questão definiremos uma imputação ou uma alocação como sendo uma redistribuição exaustiva do valor da grande coalizão que atribui ou distribui para cada jogador no mínimo a quantidade que ele garantiria para si, se não entrasse para a grande coalizão.

Representando por $\mathrm{x} \in \mathfrak{R}^{\mathrm{n}}$ o vetor de resultados e

$$
\mathbf{x}(\mathrm{K})=\sum_{\mathrm{i} \in \mathrm{K}} \mathrm{X}_{\mathrm{i}}
$$

como a somatória de todos os componentes de $\mathbf{x}$, ele será uma imputação se obedecer as seguintes propriedades:

$$
\begin{aligned}
& x_{i} \geq v(\{i\}) \text { para todo } i \in I, e \\
& \mathbf{x}(\mathbf{I})=v(\mathbf{I})
\end{aligned}
$$

Se chamarmos de $\mathrm{X}(\mathrm{v}) \subset \mathfrak{R}$ o conjunto de todas imputações de um dado jogo expresso na forma de função característica, uma solução pode ser definida como um subconjunto de $\mathbf{X}(v)$ consistindo das imputações que não forem eliminadas pelo conjunto de "filtros" ou restrições, que vamos expor logo adiante. O conjunto de restrições que regerá o processo de negociação é que definirá os diversos conceitos de solução para um jogo cooperativo. 
Nesse processo de negociação ou barganha naturalmente uma imputação x pode não satisfazer algum participante $\mathbf{k}$, nesse caso dizemos que o participante $\mathbf{k}$ tem uma objeção àquela imputação $\mathbf{x}$. Formalmente, um jogador $\mathbf{k}, \mathbf{k} \quad \mathrm{K} \quad \mathbf{I}$, tem uma objeção $(\mathbf{y}, \mathrm{K})$ à imputação $\mathbf{x}$ se

$$
\begin{aligned}
& \mathrm{y}_{\mathrm{i}}>\mathrm{x}_{\mathrm{i}} \text { para todo } \mathrm{i} \in \mathrm{K} \text {, e } \\
& \mathbf{y}(\mathrm{K}) \leq \mathrm{v}(\mathrm{K})
\end{aligned}
$$

As propriedades (8) e (9) são as mesmas propriedades que compõem a relação que chamamos de dominância. Portanto se as propriedades (8) e (9) ocorrem simultaneamente podemos também dizer que $\mathbf{y}$ domina $\mathbf{x}$, ou $\mathbf{x}$ é dominada por $\mathbf{y}$, via coalizão K. Nesse processo outra situação pode ocorrer. Os indivíduos que não entraram na coalizão $\mathrm{K}$ podem reagir e formar ou ameaçar formar uma outra coalizão L em contraposição a coalizão $K$. Nesse caso dizemos que o jogador $l \in K^{\mathrm{c}}$, onde $K^{\mathrm{c}}$ é o conjunto complementar do conjunto $K$, tem uma contra-objeção $(\mathbf{z}, L), \mathbf{l}$ L, à (y, K) de $\mathbf{k}, \mathbf{k} \quad$ L, se

$$
\begin{aligned}
& \mathrm{z}_{\mathrm{i}} \geq \mathrm{x}_{\mathrm{i}} \text { para todo } \mathrm{i} \in \mathrm{L} \backslash \mathrm{K} \\
& \mathrm{z}_{\mathrm{i}} \geq \mathrm{y}_{\mathrm{i}} \text { para todo } \mathrm{i} \in \mathrm{L} \cap \mathrm{K} \\
& \mathrm{z}(\mathrm{L}) \leq \mathrm{v}(\mathrm{L})
\end{aligned}
$$

Observe que tanto o indivíduo l quanto o indivíduo $\mathbf{k}$ estavam na imputação original x. Então o indivíduo k descontente com seus benefícios na imputação original $\mathbf{x}$ resolve fazer ou ameaçar fazer uma coalizão $\mathrm{K}$ deixando o indivíduo $\mathbf{l}$ de fora. $\mathrm{O}$ indivíduo $\mathbf{l}$ que ficou de fora não tem mais a garantia de receber o que receberia na imputação $\mathbf{x}$, para proteger seu ganho em $\mathbf{x}$ resolve fazer uma ameaça. Isto é, ele informa ao indivíduo $\mathbf{k}$ que se este resolver montar a coalizão $\mathrm{K}$, ele, o indivíduo $\mathbf{l}$, tem condições concretas para montar uma outra coalizão L, desmontando assim a coalizão $\mathrm{K}$, deixando o indivíduo $\mathbf{k}$ de fora e garantindo no mínimo o valor $\mathbf{x}_{\mathbf{1}}$ que ele tinha garantido na imputação original $\mathbf{x}$ a qual o indivíduo $\mathbf{k}$ ameaça desmontar.

Uma objeção faz sentido, ou se diz que se justifica, quando não existe nenhuma contra-objeção a ela. Dados os conceitos de imputação, objeção, contra-objeção e objeção justificada, podemos então caracterizar os principais conceitos de solução para o jogo cooperativo:

3.1 O núcleo: representado por co( $(v)$, é definido como o conjunto de todas imputações não dominadas via qualquer coalizão, ou o conjunto daquelas para as quais não existem objeções. Nesse caso escolhe-se a solução ou as soluções eliminando no curso da negociação as imputações para as quais foi apresentada qualquer objeção. 
Formalmente o núcleo pode ser representado pelo conjunto de todas imputações $\mathbf{x}$ tais que

$$
\sum_{i \in S} X_{i} \geq v(S), \forall S \subset \mathbf{I}
$$

A expressão matemática acima garante que se qualquer grupo de indivíduos $\mathrm{S}$, que faça parte do conjunto de indivíduos que compõem o jogo, resolver fazer uma coalizão, nunca obterá um valor maior do que a soma dos ganhos individuais que ele obtém na imputação x. Qualquer imputação pertencente ao núcleo é estável, no sentido de que não existe nenhuma coalizão que possua simultaneamente o estímulo e o poder de mudar o resultado do jogo.

O núcleo pode ser apresentado de outra forma. Seja $e(\mathbf{x}, K)=v(K)-\mathbf{x}(K)$ a reclamação dos membros da coalizão $K$ em relação à imputação x. Podemos então expressá-lo como o conjunto de todas imputações cujas máximas reclamações que existam contra elas são menores ou iguais a zero, isto é:

$$
\operatorname{co}(v)=\left\{\mathbf{x} \in \mathrm{X}(\mathrm{v}) \mid \max _{\mathrm{K} \subset \mathrm{I}} e(\mathbf{x}, \mathrm{K}) \leq 0\right\}
$$

3.2 Conjunto-negociação: constitui oconjunto de imputações que não possuem objeções acresentdas às imputações que possuem objeções não justificadas. Mais precisamente, constitui o conjunto de imputações que restam quando no processo de negociação uma imputação só é eliminada quando tem uma objeção e essa objeção é justificada (OWEN, 1968, pag.188).

Dada essa definição, fica claro que o conjunto-negociação deve conter o núcleo se esse existir. E se ele, o núcleo, não existir, ainda existe a possibilidade de se encontrar solução através do conjunto-negociação.

3.3 Conjunto-Solução de von Neumann-Morgenstern:. Esta solução, $N M(v)$, é representada pelo conjunto de imputações que satisfazem simultaneamente duas condições:(1). para quaisquer duas imputações pertencentes ao conjunto $\mathrm{NM}(v)$, uma não pode ser dominada pela outra; (2) qualquer imputação que não pertença ao conjunto $\mathrm{NM}(\mathrm{v})$ deve ser dominada por alguma imputação pertencente ao conjunto NM(v), (NEUMANN \& MORGENSTERN, 1972)

Uma das principais dificuldades de se utilizar o conceito de solução de Neumann \& Morgenstern é que nem a existência e nem a unicidade são garantidas. Nenhuma prova geral da existência do conjunto-estável foi feita até agora (OWEN, 1968).

3.4 Nucléolo: esse conceito, $\mathrm{Nu}(v)$, constitui um critério que seleciona as imputações de forma a minimizar a máxima queixa ou reclamação que qualquer coalizão possa ter contra elas.

O procedimento para se encontrar o nucléolo, $\mathrm{Nu}(v)$, o que significa aplicar o princípio minimax, exige que façamos uma comparação entre as diversas imputações de forma a encontrarmos aquela cuja máxima reclamação associada a ela é menor do 
que a máxima queixa associada a qualquer outra imputação. Para isso, ordenam-se as $2^{\mathrm{n}}$ coalizões contidas em $\mathbf{I}$ (incluindo o subconjunto $\mathrm{N}$ contendo todos elementos de $\mathbf{I}$ e o conjunto vazio $\phi) \mathrm{K}_{1}, \mathrm{~K}_{2}, \ldots, \mathrm{K}_{2^{\mathrm{n}}}$ e se constrói uma função $\theta(\mathbf{x})$, onde,

$$
\theta(\mathbf{x})=\left(\theta_{1}(\mathbf{x}), \theta_{2}(\mathbf{x}), \ldots, \theta_{2^{\mathrm{n}}}(\mathbf{x})\right) \in \mathfrak{R}^{2^{\mathrm{n}}} ;
$$$$
\theta_{\mathrm{j}}(\mathbf{x})=e\left(\mathbf{x}, \mathrm{K}_{\mathrm{j}}\right)
$$

$$
\theta_{j}(\mathbf{x})>\theta_{j+1}(\mathbf{x}) \text { para } j=1,2, \ldots, 2^{n-1} .
$$

Dadas duas imputações $\mathbf{x}$ e $\mathbf{x}^{\prime}$, os vetores de queixas associados respectivamente a $\theta(\mathbf{x})$ e $\theta\left(\mathbf{x}^{\prime}\right)$ podem ser comparados depois de ordenados lexicograficamente. Desta forma, podemos dizer que $\theta(\mathbf{x})$ é lexicograficamente menor que $\theta\left(\mathbf{x}^{\prime}\right)$ se $\theta_{1}(\mathbf{x})<\theta_{1}\left(\mathbf{x}^{\prime}\right)$ ou para $\mathbf{j}>1$, e $\theta_{\mathrm{i}}(\mathbf{x})=\theta_{\mathrm{i}}\left(\mathbf{x}^{\prime}\right)$ para $\mathbf{i}=1, \ldots, \mathbf{j}-1$. Essa relação pode ser representada por $\theta(\mathbf{x})<_{\mathrm{L}} \theta\left(\mathbf{x}^{\prime}\right)$. Se $\theta_{\mathrm{j}}(\mathbf{x})=\theta_{\mathrm{j}}\left(\mathbf{x}^{\prime}\right)$ para todo $\mathbf{j}$, então $\theta(\mathbf{x})={ }_{\mathrm{L}} \theta\left(\mathbf{x}^{\prime}\right)$, e $\theta(\mathbf{x}) \leq_{\mathrm{L}} \theta\left(\mathbf{x}^{\prime}\right)$ significa que $\theta(\mathbf{x})<_{\mathrm{L}} \theta\left(\mathbf{x}^{\prime}\right)$ ou $\theta(\mathbf{x})=_{\mathrm{L}} \theta\left(\mathbf{x}^{\prime}\right)$. Portanto, para um conjunto $\mathrm{X}(\mathrm{v})$, o nucléolo pode ser definido como

$$
\mathrm{Nu}(v)=\left\{\mathbf{x} \in X \mid \mathbf{x}^{\prime} \in X(v) \Rightarrow \theta(\mathbf{x}) \leq_{\mathrm{L}} \theta\left(\mathbf{x}^{\prime}\right)\right\} .
$$

Isto é, o nucléolo do jogo $v$ consiste de todas aquelas imputações $\mathbf{x}$ e $X(v)$ que possuem as menores queixas, lexicograficamente, associadas a elas. Dessa forma o nucléolo acaba se constituindo numa forma de tornar o menor possível o protesto da coalizão mais descontente.

A importância do nucléolo é que se $X(v)$ é compacto o $\mathrm{Nu}(v)$ não é vazio; se $\mathrm{X}(\mathrm{v})$ for compacto e convexo, o $\mathrm{Nu}(v)$ será constituído de um único elemento; e se o núcleo não for vazio, o nucléolo será um subconjunto do núcleo (MOULIN, 1981). Uma solução para problemas contínuos foi proposta por Kohlberg ao demonstar que é possível, em algumas situações, calcular o núcleo como a solução de um problema de programação linear (KOHLBERG, 1972a, b)

3.5 Valor de Shapley: apresentado por l. S. Shapley em 1953, no texto A value for npersons games da obra "Contritutions to the Theory of Games" organizada por H. W. Kuhn e A. W. Tucker (LUCE \& RAIFFA, 1957). Esse conceito de solução constitui um critério que distribui para cada participante do conjunto I, um valor $\Phi_{\mathrm{i}}(v)$ baseado em quatro axiomas:

1. produz uma distribuição exaustiva do valor total da grande coalizão, isto é, $\sum_{\mathrm{i} \in \mathrm{I}} \Phi_{\mathrm{i}}(\mathrm{v})=\mathrm{v}(\mathbf{I})$.

2. se um jogador i não adiciona nada mais que $v(\{i\})$ a qualquer coalizão, ele recebe somente $v(\{i\})$, isto é, $\Phi_{i}(v)=v(\{i\})$. 
3. se dois jogos são idênticos exceto quanto à ordem na qual os seus jogadores estão listados, o valor de Shapley para os jogadores são os mesmos.

4. se um jogo é formado pela adição de dois outros jogos, o valor de Shapley para o novo jogo será a soma dos valores de Shapley dos jogos iniciais.

Pode-se demonstrar (OWEN, 1968) que existe uma e somente uma função que preenche as condições estabelecidas pelos axiomas citados acima. E ela atribui a cada participante $\mathbf{i}$ do jogo $v$ um ganho ou valor dado por:

$$
\Phi_{\mathrm{i}}(v)=\sum_{\mathrm{K} \subset \mathrm{I}} \frac{(\mathrm{n}-\mathrm{k}) !(\mathrm{k}-1) !}{\mathrm{n} !}[v(\mathrm{~K})-v(\mathrm{~K} \backslash\{\mathrm{i}\})]
$$

onde $\mathbf{k}$ representa o número de indivíduos que compõem a coalizão K.

A expressão de $\Phi_{\mathrm{i}}(v)$, o valor que cada indivíduo recebe através dessa forma de redistribuição, sugere uma interpretação probabilística, onde $[v(K)-v(K \backslash\{\mathrm{i}\})]$ expressa a contribuição marginal do jogador $\mathbf{i}$ ao valor da coalizão $\mathrm{K}$ e $\frac{(\mathrm{n}-\mathrm{k}) !(\mathrm{k}-1) !}{\mathrm{n} !}$ a probabilidade de que no processo aleatório de formação da grande coalizão I, o indivíduo i seja o jogador acrescentado à coalizão consistindo, ou que consistia, dos primeiros k-1 jogadores naquela ordem aleatória. O ganho de cada jogador pode ser visto como média ponderada das contribuições que o jogador fornece a cada coalizão que ele participa, onde os pesos seriam valores que dependem do total de jogadores $\mathrm{n} e$ do número deles em cada coalizão $\mathrm{K}$, ou seja, k. O valor de Shapley pode ainda ser interpretado como um critério que atribui a cada participante do jogo a sua contribuição marginal esperada.

A fórmula do valor de Shapley pode ser deduzida diretamente através da interpretação probabilística dada acima. Suponha que os $\mathbf{n}$ jogadores combinem se encontrarem em um determinado lugar em uma determinada hora. Devido à aleatoriedade, eles chegarão em momentos diferentes no tempo. Suponha também que as ordens de chegada ( o número de permutações de $\mathbf{n}$ jogadores) têm a mesma probabilidade, isto é, $1 / \mathbf{n}$ !. Considerando que se um jogador i quando chega já encontra os membros da coalizão $[K-\{i\}]$ ele recebe a quantia $[v(K)-v(K \backslash\{i\})]$, ou seja a quantidade marginal que contribui para aquela coalizão. Logo, o valor de Shapley será o valor esperado de ganho do jogador i sob esse esquema aleatório.

Outra maneira análoga de se deduzir a mesma fórmula seria imaginarmos um evento $\mathrm{A}$, que fosse definido como: $\mathrm{A}=\{$ escolher $\mathrm{o}$ jogador $\mathbf{i}$ pertencente obrigatoriamente à coalizão de $\mathbf{k}$ elementos $\}$ e um evento $\mathrm{B}$, onde fosse definido como $\mathrm{B}=\{$ escolher os outros $\mathbf{k}-1$ elementos da coalizão entre os $\mathbf{n}-1$ jogadores restantes $\}$. A probabilidade dos eventos A e B ocorrerem simultaneamente seria 


$$
\begin{aligned}
& P(A \cap B)=P(B \backslash A) P(A)=\frac{1}{\left(\begin{array}{l}
n-1 \\
k-1
\end{array}\right)} \times \frac{1}{n}= \\
& =\frac{1}{(n-1) ! /(k-1) ![n-1-(k-1)] !} \times \frac{1}{\mathbf{n}}=\frac{(k-1) !(n-k) !}{\mathbf{n}} \text {, donde se deduz o }
\end{aligned}
$$

valor esperado para cada jogador como

$$
\Phi_{\mathrm{i}}(v)=\sum_{\mathrm{K}=1}^{\mathrm{n}} \frac{(\mathbf{n}-\mathrm{k}) !(\mathrm{k}-1) !}{\mathbf{n} !}[v(\mathrm{~K})-\mathrm{v}(\mathrm{K} \backslash\{\mathrm{i}\})]
$$

Para finalizar, observe que o valor de Shapley, $\Phi_{\mathrm{i}}(v)$ é uma imputação, dado que:

$$
\begin{aligned}
& \sum_{\mathrm{i} \in \mathrm{I}} \Phi_{\mathrm{i}}(v)=v(\mathbf{I}), \quad \mathrm{e} \\
& \Phi_{\mathrm{i}}(v) \geq v(\{\mathrm{i}\}), \forall \mathrm{i} \in \mathbf{I} .
\end{aligned}
$$

\section{Aplicação da Teoria do Jogos à Distribuição de Custo Conjunto.}

Departamentos de uma corporação industrial, cooperativas, aeronaves que utilizam um mesmo campo de aviação, ou indivíduos que têm interesse em desempenhar atividades em conjunto, por exemplo, se defrontam em geral com a questão de distribuição de custos conjuntos. A "contabilidade tradicional" sugere pelo menos cinco maneiras de se ratear custos. No entanto, todas estas maneiras apresentam arbitrariedades e só são aceitas pelas partes envolvidas quando estas estão submetidas a um poder central que as impõem. Isto é, todos estes métodos só podem ser aceitos, em geral, quando as partes não possuem a possibilidade de atuarem sozinhas ou por meios de coalizões.

A imposição destes critérios de rateios às vezes são impostos pelo governo ou por uma autoridade central que comanda as partes envolvidas. Contudo a questão toma um outro aspecto quando não existe um poder centralizado de forma que as partes livres para aceitar ou não um determinado critério de rateio podem inviabilizar um determinado projeto e, conseqüentemente, promover situações desastrosas não só para o grupo como para o resto da sociedade. A Teoria dos Jogos tem se dedicado também a esta questão e proposto algumas soluções.

A seguir apresentaremos alguns casos como forma de visualizarmos o panorama da incursão da Teoria dos Jogos nesse campo.

Suponhamos, por exemplo, que uma atividade possa ser desenvolvida por indivíduos isolados ou por uma coalizão desses indivíduos. Se a atividade for realizada isoladamente por cada indivíduo, estes incorreram em um custo particular. Se for desempenhada por um grupo ou pela comunidade como um todo haverá um ganho de escala que reduzirá consideravelmente o custo total. Ou seja, o custo total será consideravelmente menor que a soma de todos os custos individuais se os agentes agirem isoladamente. O problema com o qual nos deparamos é: como distribuir o 
ganho de escala entre os agentes que participaram em conjunto desta atividade? Em outras palavras, como distribuir o custo conjunto, dado que não existe uma forma objetiva, direta e portanto inquestionável de se ratear o custo total entre os participantes do projeto?

Uma boa ilustração do problema pode ser feita através do exemplo da construção de barragens que podem ser construídas para atender a vários objetivos. Suponhamos por exemplo que o custo da construção de uma barragem com o propósito de fornecer água para irrigação seja de 10 bilhões de reais, enquanto que o custo de uma barragem para a contenção de enchentes seja de 8 bilhões de reais. Pode-se construir uma barragem que atenda a ambos propósitos por 15 bihões de reais. Neste caso existe um ganho de 3 bilhões de reais se a barragem for construída conjuntamente e esse benefício deve ser distribuído entre os participantes do empreendimento através do rateio do custo total de 15 bilhões de reais. Como existem apenas dois participantes, a questão torna-se bem simples. Basta que, como condição necessária, os custos sejam distribuídos de forma que nenhum participante se sinta subsidiando o outro. Isto é, a distribuição de custos não pode impor que um agente subsidie o outro. Formalmente, se denominarmos de $C(\{i\}), i=1,2$, o custo de construção da barragem para atender $o$ objetivo do agente i e $\mathrm{R}(\{\mathrm{i}\})$ a sua respectiva contribuição, a condição para que não haja imposição de subsídio será então:

$$
\mathrm{R}(\{\mathrm{i}\}) \leq \mathrm{C}(\{\mathrm{i}\}) \mathrm{i}=1,2 .
$$

Quando a questão envolve apenas dois agentes torna-se mais simples porque só existe uma possibilidade de coalizão, ou seja, a coalizão dos dois agentes. Quando existem mais de dois agentes, a possibilidade de coalizões obviamente aumenta e pode ser que a condição posta acima não baste como mínimo necessário para que a distribuição de custo seja aceitável pelos envolvidos. Neste caso, temos que levar em conta não só os agentes, mas as suas possíveis coalizões. Conseqüentemente, para que nenhum agente e/ou nenhuma coalizão se sinta subsidiando alguém, torna-se necessário que a distribuição de custos satisfaça a seguinte condição:

$$
\mathrm{R}(\mathrm{S}) \leq \mathrm{C}(\mathrm{S}), \quad \text { onde } \quad \mathrm{P}(\mathrm{S})=\sum_{\mathrm{i} \in \mathrm{S}} \mathrm{R}(\{\mathrm{i}\}) \text { para todo } \mathrm{S} \subseteq \mathbf{I} \text {, }
$$

onde I é o conjunto de todos participantes e S representa qualquer coalizão em I, incluindo tanto o próprio I como a "coalizão" de um elemento.

Na forma em que foi posta a questão podemos facilmente transportá-la para a Teoria dos Jogos. Assim a distribuição de custo entre os agentes pode ser feita caraterizando a questão como um jogo cooperativo com pagamentos colaterais. A função de custo determina a função característica de um "jogo de distribuição de custos" subaditiva, em vez de superaditiva, enquanto o vetor de receitas, ou contribuições, representa o vetor de resultados. Se o vetor de resultados pode ser representado como: 


$$
\sum_{i \in S} R(\{i\})=C(I) .
$$

então pode ser visto como uma imputação. Se este vetor de resultado além de atender a condição (27) satisfaz também a condição (26) então ele se encaixa perfeitamente no conceito de núcleo, dado que nenhuma destas imputações pode ser bloqueada por qualquer coalizão de jogadores, no sentido de que seria mais vantajoso (ou menos custoso) contribuir com R(S) do que construir a barragem através da coalizão $S$, a qual teria um custo $\mathrm{C}(\mathrm{S}) \geq \mathrm{R}(\mathrm{S})$.

Vejamos o seguinte exemplo hipotético. Suponhamos que uma barragem possa ser construída para três objetivos diferentes: produção de energia elétrica, controle de en-chentes e irrigação. Temos agora 3 agentes indexados de acordo com seus interesses: i = 1 representa o agente interessado na produção de energia elétrica; i =2, o agente interessado no controle de enchentes; e i = 3, o agente interessado na contenção de água para irrigação. Suponhamos que o custo de construção da barragem para atender somente a produção de energia elétrica seja $C(\{1\})=10$ bilhões de reais; para o controle de enchentes seja $C(\{2\})=8$ bilhões de reais; e para contenção d'água para prover irrigação seja $C(\{3\})=7$ bilhões de reais. No entanto, eles podem se coligar livremente para a construção da barragem. Neste caso, suponhamos que o custo para a construção para atender simultaneamente a produção de energia elétrica e o controle de enchentes será $C(\{1,2\})=15$ bilhões de reais; para atender a produção de energia e irrigação será $C(\{1,3\})=14$ bilhões de reais; para atender ao controle de enchentes e irrigação será $C(\{2,3\})=16$ bilhões de reais; e finalmente para atender aos três objetivos será $C(\{1,2,3\})=20$ bilhões de reais. Qual deverá ser a distribuição de custos entre os 3 agentes para que construam uma só barragem para atender conjuntamente aos três objetivos, dado que dessa forma teriam um ganho de 5 bilhões de reais em relação ao custo que teriam se as barragem fossem construídas individualmente?

A questão se resume em encontrar o núcleo do jogo cooperativo caracterizado pela situação. Portanto, bastaria encontrar um conjunto de imputações que não sejam dominadas via qualquer outra coalizão. Ou seja, basta encontrarmos uma distribuição de custos definida pelos valores $R_{i}, i=1,2,3$, tais que:

$$
\sum \mathrm{R}_{\mathrm{i}}=\mathrm{C}(\{1,2,3\}) \mathrm{e}
$$

Para tanto bastaria construir um programa computacional que determinasse os valores de $\mathrm{R}_{1}, \mathrm{R}_{2}$, e $\mathrm{R}_{3}$ tal que:

$$
\mathrm{P}_{1} \leq 10
$$

$$
\mathrm{P}_{2} \leq 8
$$

$$
\mathrm{P}_{3} \leq 7
$$




$$
\begin{aligned}
& \mathrm{P}_{1}+\mathrm{P}_{2} \leq 15 \\
& \mathrm{P}_{1}+\mathrm{P}_{3} \leq 14 \\
& \mathrm{P}_{2}+\mathrm{P}_{3} \leq 16 \\
& \mathrm{P}_{1}+\mathrm{P}_{2}+\mathrm{P}_{3}=20
\end{aligned}
$$

Supondo que a distribuição de custos se faça apenas em forma de números inteiros teríamos um núcleo de nove elementos, ou seja: $(5,8,7),(6,8,6),(6,7,7)$, $(7,8,5),(7,7,6),(7,6,7),(8,7,5),(8,6,6),(9,6,5)$. Estas seriam as possíveis distribuições de custos de forma a que a barragem possa ser construída para atender os três objetivos em conjunto e sem que haja subsídios cruzados. Porém, como existe mais de uma distribuição possível do custo total, poderia se aplicar tanto o conceito de solução chamado nucléolo como o valor de Shapley para auxiliar a busca de uma solução única.

O nucléolo não daria uma distribuição pertencente ao núcleo de forma a minimizar as queixas de qualquer outra coalizão contra ela. Aplicando o processo de maximização lexicográfica encontramos o nucléolo igual a $(7,7,6)$. Aplicando o conceito de valor de Shapley, onde implicitamente se considera o resultado como um distribuição justa, dado que cada agente recebe o valor esperado de sua contribuição marginal, encontramos o mesmo vetor $(7,7,6)$. Portanto, uma razoável proposta de distribuição de custo para esta questão hipotética deve ser $(7,7,6)$, porque ela está no núcleo; representa no núcleo a distribuição que minimiza as reclamações das outras possíveis coalizões; e diz respeito a uma distribuição onde cada agente é sobrecarregado proporcionalmente à sua contribuição ao ganho de 5 bilhões de reais na construção da barragem para atender os três objetivos em conjunto.

Não existe obviamente forma de se obrigar os agentes a aceitarem qualquer distribuição contida no núcleo. Mas todos preferem estas a qualquer outra alternativa . A distribuição $(7,7,6)$ pode ser usada pelo mediador da questão que por sua vez pode usar como forma de persuasão o conceito de nucléolo e do valor de Shapley simultaneamente.

Uma outra aplicação muito evidenciada na literatura foi proposta por Martin SHUBICK (1962). A "contabilidade" apresenta vários critérios de rateio de custos conjuntos e todos eles são criticados por Shubick pelo fato de não apresentarem formas descentralizadas de incentivos, aos diversos centros de produção ou departamentos de uma corporação, para a inovação.

Segundo SHUBICK (1962) o objetivo de uma boa gerência seria projetar um sistema de premiação para aqueles que assumem riscos no processo de tomada de decisão de tal maneira que os prêmios individuais tenham uma correlação positiva com o valor do resultado da decisão para a organização. Em uma corporação se o poder de decisão deve ser delegado, é preferivel termos uma organização projetada para encorajar a iniciativa dos centros de decisão. Uma forma de promover isto seria termos uma estrutura de premiação projetada de forma que a seleção das melhores alternativas 
para o tomador de decisão individual coincida sempre com aquelas que são melhores para a organização como um todo. Este requisito não está garantido pelos métodos de distribuição de custo conjunto convencionais oferecidos pela "contabilidade". Por exemplo, a gerência de uma fábrica pode muito bem ter conhecimento de uma mudança, que por sua vez pode ter o efeito de aumentar o lucro da corporação, no entanto, essa decisão implica em diminuir o tamanho de sua fábrica e reduzir o lucro atribuído a ela pelo sistema contábil. Se seu sucesso e renda são determinados pelos lucros contábeis atribuídos à sua fábrica, ou departamento, provavelmente ela não ficará interessada em tomar essa decisão, a qual seria ótima para a corporação.

A lucratividade de uma corporação pode ser vista como dependente da soma dos lucros conjuntos que, por seu turno, podem ser obtidos pela coordenação ótima de todas unidades, por exemplo, fábricas, departamentos, etc... E como existe um custo administrativo que é um custo conjunto, ele deve ser rateado entre todos os centros de decisão, de forma que o interesse privado de cada centro de decisão coincida com o interesse da organização. Esta situação configura um jogo cooperativo onde os agentes são os centros de decisão que compõem a corporação.

Outro exemplo importante de distribuição de custo conjunto foi apresentado por SUZUKI \& NAKAYAMA (1976) que aplicaram a Teoria dos Jogos à questão de exploração conjunta de recursos naturais por um grupo de indivíduos.

A motivação dos autores na aplicação da Teoria dos Jogos à questão se resume no fato de que, à medida que se aumenta a demanda por um recurso natural, como a água, por exemplo, aumenta-se também com muita intensidade os custos de exploração para o fornecimento deste acréscimo de demanda, principalmente onde a questão foi tratada, ou seja, no Japão. Este custo enorme, por sua vez, exige que a exploração para o fornecimento adicional destes recursos seja desenvolvido por alguma forma de cooperação entre os interessados, constituindo assim uma espécie de jogo cooperativo. E a solução proposta para esse jogo contempla um conceito de justiça, segundo o qual cada participante deve receber os custos e benefícios de acordo com a contribuição do mesmo.

Em síntese, a questão é a seguinte: no Japão, os recursos hidráulicos encontrados na região em estudo são explorados por dois agentes distintos: (1) as cooperativas agrícolas que consideram a oferta de água existente como adequada, ou mais que adequadas, para suas necessidades de irrigação e (2) os serviços de distribuição d'água das cidades, cuja necessidade correntes ou futuras de abastecimento não são atendidas pelas fontes existentes.

Desta forma qualquer exploração adicional de recursos hidráulicos para atender às necessidades crescentes das cidades, na região, exigirá alguma espécie de acordo entre esses dois tipos de usuários do potencial hidráulico. Se considerarmos $N=\{1,2$, ..., n $\}$ o conjunto de todos os agentes envolvidos, podemos particioná-lo em dois subconjuntos distintos: a letra $\mathrm{A}$, representando um subconjunto de $\mathrm{N}$ contendo todas as associações agrícolas; e a letra $\mathrm{B}$, também como o subconjunto de $\mathrm{N}$ contendo todos serviços de distribuição de água das cidades.

Cada cidade i contida em B requer uma quantidade adicional anual de água. Seja $\delta_{\mathrm{i}}$ a quantidade adicional anual demandada pelo o agente i. Logo $\delta_{\mathrm{i}}=0$ se $\mathrm{i} \in \mathrm{A}$. 
Tecnologicamente, existem duas formas pelas quais uma cidade i pode adquirir a quantidade de água adicional $\delta_{i}$ que ela precisa: (1) construindo uma barragem, com ou sem a cooperação das outras cidades; ou (2) fazendo um acordo, ou uma coalizão, com as associações agrícolas para o desvio direto da água para a cidade. Uma cidade ainda pode optar por uma combinação das alternativas (1) e (2) para atender às suas necessidades. Naturalmente, essas opções custam dinheiro, mas o motivo crucial que levaria os agentes a formarem coalizões está no fato de que o custo envolvido em atender suas necessidades através de uma coalizão deve ser menor do que a soma dos custos quando procuram atender suas necessidades atuando sozinhos. Uma vez verificada esta condição, torna-se necessário somente que seja garantido aos membros da coalizão que o custo poupado possa ser distribuído entre eles de certa forma "justa". Os autores formularam a questão como um jogo cooperativo e propuseram o conceito de solução nucléolo como forma "justa" de distribuição do custo conjunto poupado.

Seja S qualquer coalizão de cidades e/ou associações agrícolas, ou seja, S é um subconjunto de N. Seja v(S) o mínimo custo para se atender a necessidade adicional de água de todos membros de $S$, supondo nenhuma cooperação dos agentes que ficam fora de $S$. Por exemplo, se $S \cap B=\phi$, isto é, se $S$ é uma coalizão de associações agrícolas então $v(S)=0$, dado que as associações agrícolas não possuem nenhuma necessidade adicional de água. Se $S \cap A=\phi$, isto é, $S$ é uma coalizão de cidades, então a alternativa tecnológica (1) citada acima é a única opção disponível a $S$, na medida em que a alternativa (2) requer a cooperação das associações agrícolas que estão fora de $S$. Logo se $S \cap A=\phi$, então $v(S)$ deve ser o custo de construção de uma barragem capaz de atender as necessidades adicionais de água de todas as cidades em $\mathrm{S}$.

Se S é uma coalizão contendo tanto os serviços de distribuição de água das cidades como as associações agrícolas, então v(S), o mínimo custo para se atender as necessidades de água das cidades em S, deve ser a soma de três custos separados: (1) o custo de uma barragem capaz de atender parte da demanda adicional de água dos membros de S; (2) o custo do desvio de água das associações agrícolas para a cidade afim de atender a demanda remanescente de água adicional, e finalmente (3) o custo para compensar as associações agrícolas pelo decréscimo na produção agrícola devido o desvio direto da água. Por exemplo, se $S=\{1,2,3,4,5\}$, onde os agentes 1 e 2 são associações agrícolas e os agentes 3, 4 e 5 são serviços de distribuição de água das cidades, então os membros de S podem achar, supondo nenhuma cooperação dos jogadores que estão fora de $\mathrm{S}$, que a forma mais barata de atender suas necessidades adicionais de água seja construir uma barragem capaz de atender totalmente as necessidades da cidade 5 e atender $50 \%$ da necessidade adicional de água da cidade 4 e então desviar água das associações agrícolas 1 e 2 para atender a necessidade da cidade 3 e $50 \%$ da necessidade adicional remanescente de água da cidade 4 . Mas se este desvio usa bastante água do agente 2, a ponto de prejudicar sua produção agrícola, então o agente 2 terá de ser recompensado pelo seu prejuízo.

A determinação de $v(S)$ para cada possível coalizão $S$ não é sempre fácil. Nos casos onde a forma da função de custos das alternativas (1), (2) e (3) são conhecidas ou podem ser aproximadas, a determinação de $v(S)$ para qualquer dado $S$ constitui um problema de programação matemática. Contudo uma vez determinado v(S) para cada 
coalizão $\mathrm{S} \subseteq \mathrm{N}$, o problema pode ser caracterizado como um jogo cooperativo de npessoas, e a distribuição do custo conjunto pode ser feita através do nucléolo.

SUZUKI \& NAKAYAMA (1976) aplicaram o modelo de exploração cooperativa de recursos hídricos descrito acima para as cidades Kanagawa, Yokohama, Kawasaki e as Associações Agrícolas dos rios Sakawa e Sagami, no qual se supôs que a barragem seria construída sobre o rio Sakawa e o desvio de água do uso agrícola para o serviço de distribuição d'água da cidade seria realizado na bacia dos dois rios.

LITTLECHILD \& THOMPSON (1977) também desenvolveram aplicações práti-cas da Teoria dos Jogos. E entre elas cabe destacar a análise de custo de uso do aeropor-to de Birmingham, (Ing.). Para tanto, eles construíram um jogo com 13572 agen-tes no qual o núcleo, devido à economia de escala na construção das pistas de decola-gem, se apresentou não-vazio. A seguir calcularam o nucléolo, o qual, no caso, determi-na a taxa para todas aeronaves de diferentes tamanhos. Observando as taxas pagas entre 1968 e 1969, eles concluíram que as aeronaves menores subsidiaram as aeronaves maiores.

Um problema semelhante se verifica quando as empresas quando adotam conjunta-mente investimentos que realizam sinergia ou de custos produzindo uma receita líquida maior. A alocação dos custos financeiros, nestes casos, é em geral arbitrária. CALLEN (1978) mostra como o conceito de valor de Shapley pode ser utilizado para distirbuição de custos financeiros de projetos intedenpendentes de forma mais consistente.

\section{Considerações finais.}

A Teoria dos Jogos, apesar de curtos momentos de estagnação, nunca deixou de progredir. Sua história constitui um processo de desenvolvimento de novos conceitos e técnicas. No entanto chega-se a um determinado momento de desenvolvimento e de especialização destas técnicas onde, aparentemente, rompe-se o elo entre conceito e realidade. De forma que essa metodologia, que possue um elo tão orgânico em relação ao comportamento humano pode, devido obviamente ao alto grau necessário de abstração das novas técnicas, ser equivocadamente vista como uma mera abstração e sem qualquer ligação com o mundo real. A sistematização que apresentamos neste texto torna se importante porque, primerio, reúne os conceitos fundamentais de onde se deriva todos os conceitos modernos. E segundo, por serem conceitos básicos, permitem facilmente se verificar sua organicidade com a prática. Em outras palavras, esta sistematização nos permite visualizar facilmente os pressuposto da metodologia, qual seja o de que os homens estabelecem relações para satisfazerem seus interesses. Algo que muitas teorias acabam, premeditadamente ou não, camuflando.

A Teoria dos Jogos tem promovido contribuições nos mais distinto ramos do conhecimento humano. Apresentamos, de forma ilustrativas, alguns casos onde questões típicas da Engenharia de Produção podem ser tratada de forma exemplar pela Teoria dos Jogos tal como a questão da Distribuição de Custos Conjuntos.

\section{REFERÊNCIAS BIBLIOGRÁFICAS.}


ARROW, K. J.: Social Choice and Individual Values (2a ed). Wiley, New York, 1965. BINMORE, K.: Fun and Game.: D. C. Heath and Company, Toronto, 1992.

CALLEN, J. L.: "Financial Cost Allocations: A Game-Theoretic Approach". The Account Review, Vol. LIII, No. 2, April, 1978

FARREL, J. \& MASKIN, E: "Renegotiation in Repeated Games". Games and Economic Behavior, 1(4),. Dec. 1989.

FIGUEIREDO, R.: A modelagem do Conflito e a Teoria dos Jogos: fundamentos econômicos e desdobramentos filosóficos.. Tese de doutorado. 319p, IEI/UFRJ, 1993.

FRIEDMAN, J. W.: Oligopoly and the Theory of Games. North-Holland, Amst. 1977.

FUDENBERG, D. \& TIROLE, J. "Perfect Bayesiano Equilíbrium and Sequential Equilibrium”. Journal of Economic Theory, 53:236-60, 1991

HURVICZ, L.: “The Design of Mechanisms for Resource Allocation”. American Economic Review, vol. 63, n 2, May 1973.

JACQUEMIN, A.: The new industrial organization. MIT Pres, 1987.

KOLHBERG, E.: "On the nucleolus of a Characteristic Function Game”. SIAM J. Appl. Math. vol. 20, $\mathrm{n}^{\circ}$ 1, Jan. 1972 b.

KOLHBERG, E.: “The nucleolus as a solution of a minimization problem”. SIAM J. Appl. Math. vol. 23, $\mathrm{n}^{\mathrm{0}}$ 1, Julho, 1972a.

KREPS, D. A course in microeconomic theory. Princeton: Princeton University Press, 1990.

LITTLECHILD, S. C. \& THOMPSON, G. F. “Aircraft landing fees: A Game Theory” Approach. Bell Journal of Economics, vol. 8, n 1, spring 1977.

LUCE, R. D. \& RAIFFA, H. Games and Decisions. Wiley \& Sons, New York, 1957.

NASH, J. F.: “Non-cooperative Games”. Annals of Mathematics, n 54,1951.

ORTEGA, José Antonio. O encontro de von Neumann com a Teoria Econômica. (mimeo). Tese de Livre-Docência apresentada ao IEI, U.F/R.J,1988

OWEN, G.: Game Theory. Saunders, Philadelphia, 1968.

SATTERTHWAITE, M. A. "Strategy-Proofness and Arrows's Conditions: Existence and Correspondence Theorems for Voting Procedures and Social Welfare Functions”. J. Econ. Theory, April, 1975, 10(2), p. 187-217.

SCHOTTER, A. SCHWODIAUER, G. "Economics and Theory of Games". Journal of Economic Literature, vol. XVIII, Jun. 1980.

SHUBIK, M.: "Incentives, Descentralized Control, the Assignment of Joint Cost and Internal Price”. Management Science, vol. 8, nº 2, April 1962.

SHUBIK, M.: Game Theory in Social Sciences. MIT Press, Massachusetts, 1987.

SUZUKI, M. \& NAKAIAMA, M.: “The Cost Assignment of the Cooperative Water Resource Development: A Game Theoretical Approach”. M. Sci. 22(10) Jul. 1976.

TIROLE, J.: The theory of industrial organization. MIT Press, Cambridge, 1988.

von NEUMANN, J. \& MORGENSTERN, O.: Theory of Games and Economic Behavior. Princeton University Press, Princeton, 1972. 


\title{
GAME THEORY: CONCEPTS, MATHEMATICAL FORMALIZATION AND APPLICATIONS TO THE ASSIGNMENT OF JOINT COST.
}

\begin{abstract}
This paper presents the basic concepts of Game Theory and the mathematical formalizations of classic concepts of game solutions. We surveyed the fundamental concepts from where we derive the concepts of game solutions that can be found in the modern papers. We feature the concepts to the game named cooperative-games by concepts of objection and counterobjection to show that they can be understood as a negotiation process. In addition, we show how the Game Theory can be applied to the problem of the assignment of joint cost.
\end{abstract}

Key-words: interdependent decisions, modelling of the conflict, game, mathematical modelling. 